\title{
Stellar Coronal Spectroscopy with the Chandra HETGS
}

\author{
David P. Huenemoerder, Bram Boroson, Norbert S. Schulz, \\ \& Claude R. Canizares \\ Massachusetts Institute of Technology, \\ 77 Vassar St., Cambridge, MA 02139 USA
}

Derek L. Buzasi, Heather L. Preston

U.S. Air Force Academy, Colorado Springs, CO 80840-6254 USA

Joel H. Kastner

Rochester Institute of Technology, Rochester, NY 14623 USA

\begin{abstract}
.
Spectroscopy with the Chandra High Energy Transmission Grating Spectrometer (HETGS) provides details on X-ray emission and activity from young and cool stars through resolution of emission lines from a variety of ions. We are beginning to see trends in activity regarding abundances, emission measures, and variability. Here we contrast spectra of TV Crt, a weak-lined T Tauri star (WTT), with TW Hya, a Classical T Tauri star (CTT). TV Crt has a spectrum more like magnetic activity driven coronae, relative to the TW Hya spectrum, which we have interpreted as due to accretion-produced X-rays. We have also observed the long period system, IM Pegasi to search for rotational modulation, and to compare activity in a long period active binary to shorter period systems and to the pre-main sequence stars. We detected no rotational modulation, but did see long-duration flares.
\end{abstract}

\section{Introduction}

$\mathrm{X}$-ray emission is ubiquitous among late-type and pre-main sequence (PMS) stars, as has been amply demonstrated by imaging and low-resolution X-ray observatories (Feigelson \& Montmerle 1999). With the advent of the Chandra transmission-grating and the XMM-Newton reflection-grating spectrometers, we can now probe the nature of the X-ray emission in detail through high-resolution line and continuum diagnostics. Early Chandra results confirmed some of the abundance anomalies derived from low-resolution imaging spectra and also unambiguously confirmed that few-component temperature models are generally not adequate. Much effort is now being spent to survey and analyze stellar Xray spectra for a range of intrinsic emission conditions provided by evolutionary state, rotational periods, and activity level. We can derive temperature distributions and elemental abundances from line-based emission measure analysis 
under assumptions regarding collisional ionization equilibrium, ionization balance, and uniformity of emitting plasma. Solutions are not unique even under these conditions, but they are a necessary starting point.

Here we present preliminary results for TV Crt (HD 98800), a WTT, and IM Pegasi (HD 216489, HR 8703), a long period RS CVn binary.

\section{Pre-Main Sequence X-Ray Emission}

The TW Hya Association (TWA) is an association of about 30 (Zuckerman et al. 2001) PMS stars at about 50 pc from the Sun (Kastner et al. 1997) and far from star forming clouds. The TWA has provided new insight into the process of star and planet formation (see for examples Jayawardhana et al. 1998, Jura et al. 1998, Holland et al. 1998).

We observed the WTT star system, TV Crt (HD 98800), a TWA member, to compare its X-ray spectrum with that of the actively accreting TW Hya. TV Crt is a hierarchical quadruple and is one of the best studied examples of a solar mass star-disk system (Soderblom et al. 1998). Two resolved sources, TV Crt- $A$ and $-B$, are visible in the Chandra zero-order image, with diskless component $A$ being about three times brighter than $B$ (Kastner et al. 2003). Component $A$ also flared during our observation. In the dispersed spectrum, we cannot separate the $A \& B$ due to their proximity ( $<1$ arcsec), given the spectrograph's astigmatism in the spatial dimension.

The first high-resolution X-ray spectrum of a low-mass CTT, TW Hya, showed dramatic differences from other stellar coronal spectra obtained to date: a very cool, sharply peaked temperature distribution, and line ratios indicative of high densities. Abundances were similar to coronae, but more extreme: very weak iron, and very strong neon. Optical and IR observations of TW Hya showed that its disk is nearly face-on (Krist et al. 2000, Muzerolle et al. 2000, Trilling et al. 2001). From this and the peculiar X-ray line properties, we attributed the X-ray emission to accretion rather than coronal emission from some form of rotational dynamo-driven magnetic activity. Kastner et al. (2002) described the results in detail.

The difference between TV Crt and TW Hya in the helium-like, densitysensitive, triplets of O VII and Ne IX is obvious even in a casual inspection of the spectra. Above a critical density, collisional excitation dominates recombination of the forbidden line $(f)$ and increases the strength of the intercombination line $(i)$. (The ratio can also be affected by ultraviolet photo-excitation which is significant in systems with $\mathrm{O}$ or B stars, but which we do not believe to be significant in TW Hya.) The $f / i$ ratios for TV Crt are at or near the low density limit, more like the typical coronal spectra of active binaries (presumably magnetic-dynamo driven). Figure 1 shows the Ne IX and O VII regions for each system.

Our preliminary emission measure modeling shows that TV Crt's plasma is hotter than that of TW Hya (Figure 2). Both systems display a cool component at $\log T=6.5$, but the emission measure distribution of TV Crt is flatter and remains high until about 7.0 before declining. The abundance anomalies deduced from the TV Crt spectrum are not as extreme as in the case of TW Hya, in which $\mathrm{Fe}$ and $\mathrm{Ne}$ ratios to Solar were about 0.1 and 2.0; in TV Crt they are 

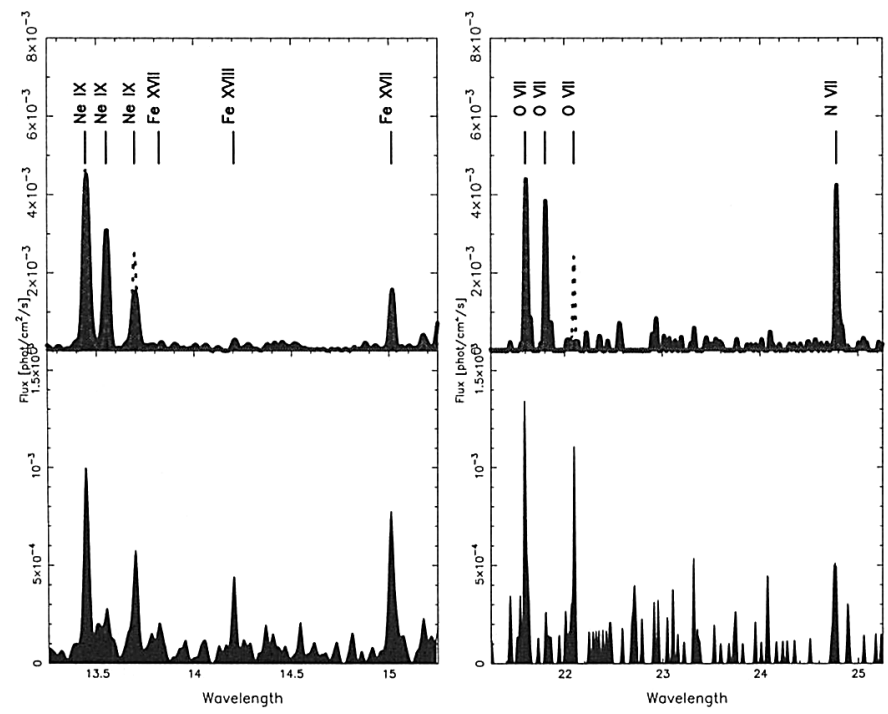

Figure 1. On the left is the Ne IX-triplet region, with resonance, intercombination, and forbidden lines at 13.447, 13.552, and $13.699 \AA$ $(r, i, f)$. The top graph is TW Hya, and the bottom, TV Crt. Note the relative strengths of the $f$ and $i$ lines. On the right is the O VII triplet region $(21.6,21.8$, and $22.1 \AA)$. The difference is extreme: no detectable $f$ line in TW Hya whereas in TV Crt the $r$ and $f$ are of comparable intensity and the $i$ line is only a marginal detection (the gray, dotted curve is the low-density theoretical spectrum).

about 0.2 and 1.0, respectively. Further analysis requires iteration using the derived plasma model to define a physical continuum, then re-measuring lines, and re-deriving the emission measure and abundances until consistent.

\section{Magnetically Active Binaries}

Solar activity has long been used as a paradigm for the interpretation of activity in cool stars which are presumed to possess a magnetic dynamo based on determinations of magnetic fields and activity cycles (Saar 1996, Baliunas et al. 1996). The existence of solar-like features such as spots and flares in addition to correlations between rotation rate and X-ray luminosity (Pallavicini 1989) lends strong support to the notion of stellar dynamos, but details of solar and stellar dynamos remain obscure (see, e.g., Charbonneau and MacGregor, 1997 for a discussion of dynamo theory).

One goal of research on stellar activity is to determine the underlying coronal heating mechanism, which is not directly observable. Fundamental observable quantities must be derived for a range of stellar parameters in order to provide the basis for a theoretical understanding. Our immediate goals are to use the phased and total X-ray spectra of a long-period active binary to address several issues: determine the temperature distribution; determine abundances; 
probe the geometry via rotational modulation and density diagnostics; monitor for flares; and compare to other stars vs. evolutionary state and environment.

IM Pegasi, a 24d period, K2 III RS CVn binary, is one of a very few RS CVn binaries to have shown rotational modulation in both lower and upper atmospheric emission (Huenemoerder, Ramsey, \& Buzasi 1990). We monitored IM Peg with the HETGS during two rotations with eight observations of $25 \mathrm{ks}$ each. While there were slow flux modulations, they did not repeat with rotational phase. Hence, we presume they are signatures of long-duration flares, especially since the amplitude of variability, though small, was largest at short wavelengths. We are in the process of performing line-based, iterative emission measure analysis vs. flux level. A preliminary emission measure, integrated over the full $200 \mathrm{ks}$ is shown in Figure 2. The emission measure and abundance distributions for IM Peg are quite similar to those of the 2-day period RS CVn, AR Lac (Huenemoerder et al. 2003). The hotter peak in the IM Peg distri-

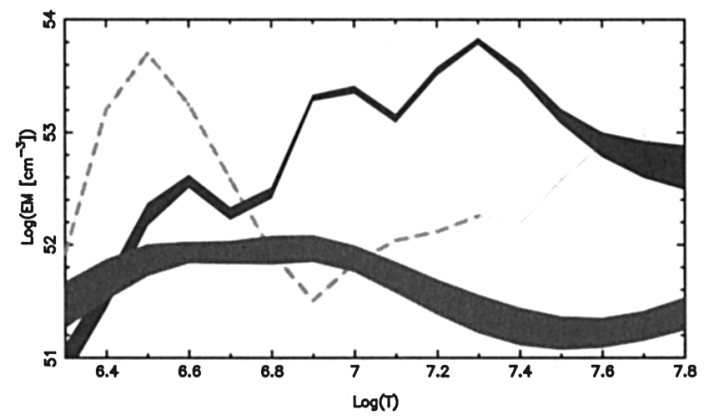

Figure 2. The upper filled curve is a differential emission measure fit to IM Peg, and the lower to TV Crt. The breadth of the band is the one sigma dispersion from a Monte Carlo calculation in which each fit was obtained by perturbing the measured fluxes according to their statistical uncertainties. The dotted line is for TW Hya, adapted from Kastner et al. (2002), which is an upper limit above $\log T \sim 7.3$.

bution (near $\log T=7.3$ ) is probably due to flaring, since we have seen direct correlation of this peak with flares in other stars (Huenemoerder, Canizares, \& Schulz 2001). Note the lack of such a peak in the TV Crt curve, and the cool, sharply peaked distribution of TW Hya.

\section{Conclusions}

The comparison of three spectra from an actively accreting system, a young but disk-free system, and a "traditional" magnetically active corona shows a suggestive progression from accretion to dynamo driven X-rays. The temperature and density may be clues to differences in X-ray origin. But there are also similarities requiring explanation: the elemental abundance trends, and the occurrence of flares. A good sample of high-resolution X-ray spectra of RS CVn binaries and flare stars exists, but many more PMS spectra are needed to help resolve these questions. 
Acknowledgments. Support for this work was provided by NASA contract NAS8-01129, SAO SV1-61010 (CXC/MIT), and NASA-Chandra Award Number G02-3005A issued by the CXC, which is operated by SAO for and on behalf of NASA under contract NAS8-39073. We also thank the Air Force Office of Scientific Research for their support.

\section{References}

Baliunas, S. L., Nesme-Ribes, E., Sokoloff, D., \& Soon, W. H., 1996, ApJ, 460, 848

Charbonneau, P., \& MacGregor, K. B., 1997, ApJ, 486, 502

Feigelson, E. D., \& Montmerle, T., 1999, ARA\&A, 37, 363

Holland, W. S., et al., 1998, Nature, 392, 788

Huenemoerder, D. P., Canizares, C. R., Drake, J. J., \& Sanz-Forcada, J., 2003, ApJ preprint doi:10.1086/377490

Huenemoerder, D. P., Canizares, C. R., \& Schulz, N. S., 2001, ApJ, 559, 1135

Huenemoerder, D. P., Ramsey, L. W., \& Buzasi, D. L., 1990, ApJ, 350, 763

Jayawardhana, R., Fisher, S., Hartmann, L., Telesco, C., Pina, R., \& Fazio, G., 1998, ApJ, 503, L79+

Jura, M., Malkan, M., White, R., Telesco, C., Pina, R., \& Fisher, R. S., 1998, ApJ, 505, 897

Kastner, J. H., Huenemoerder, D. P., Schulz, N. S., Canizares, C. R., Li, J., \& Weintraub, D. A., 2003, ApJ (submitted)

Kastner, J. H., Huenemoerder, D. P., Schulz, N. S., Canizares, C. R., \& Weintraub, D. A., 2002, ApJ, 567, 434

Kastner, J. H., Zuckerman, B., Weintraub, D. A., \& Forveille, T., 1997, Science, 277,67

Krist, J. E., Stapelfeldt, K. R., Ménard, F., Padgett, D. L., \& Burrows, C. J., 2000, ApJ, 538, 793

Muzerolle, J., Calvet, N., Briceño, C., Hartmann, L., \& Hillenbrand, L., 2000, ApJ, 535, L47

Pallavicini, R., 1989, A\&A Rev., 1, 177

Saar, S. H., 1996, in IAU Symp. 176: Stellar Surface Structure, 237

Soderblom, D. R., et al., 1998, ApJ, 498, 385

Trilling, D. E., Koerner, D. W., Barnes, J. W., Ftaclas, C., \& Brown, R. H., 2001, ApJ, 552, L151

Zuckerman, B., Webb, R. A., Schwartz, M., \& Becklin, E. E., 2001, ApJ, 549, L233 